\title{
Hypersecretion of vaccine antigen outer membrane lipoprotein A in Corynebacterium glutamicum through high-throughput based development process
}

\author{
Manman Sun ${ }^{1}$, Xiong Gao ${ }^{2}$, Rodrigo Ledesma-Amaro ${ }^{3}, \mathrm{An} \mathrm{Li}^{1}$, Rongbing Wang ${ }^{4}$, Jiangqi \\ $\mathrm{Nie}^{1}$, Pei Zheng ${ }^{5}$, Yankun Yang ${ }^{1}$, zhonghu bai ${ }^{6}$, and xiuxia liu ${ }^{6}$ \\ ${ }^{1}$ Jiangnan University \\ ${ }^{2}$ The Hong Kong University of Science and Technology \\ ${ }^{3}$ Department of Bioengineering \\ ${ }^{4}$ University of Turku \\ 5 Tecon Biology CO.Ltd \\ ${ }^{6}$ National Engineering Laboratory for Cereal Fermentauition Technology
}

December 21, 2021

\begin{abstract}
Outer membrane lipoprotein $\mathrm{A}(\mathrm{OmlA})$ is a vaccine antigen against porcine contagious pleuropneumonia (PCP), a disease severely affecting the swine industry. Here, we aimed to systematically potentiate the secretory production of OmlA in Corynebacterium glutamicum (C. glutamicum), a widely used microorganism in the food industry, by establishing a holistic development process based on our high-throughput culture platform. The expression patterns, expression element combinations, medium composition, and induction conditions were comprehensively screened or optimized in microwell plates (MWPs), followed by fermentation parameter optimization in a $4 \times 1 \mathrm{~L}$ parallel fermentation system (CUBER4). An unprecedented yield of $1.01 \mathrm{~g} / \mathrm{L}$ OmlA was ultimately achieved in a 5-L bioreactor following the scaling-up strategy of fixed oxygen mass transfer coefficient $(\mathrm{kLa})$, and the produced OmlA antigen showed well-protective immunity against Actinobacillus pleuropneumoniae challenge. This result provides a rapid and reliable pipeline to achieve the hyper-production of OmlA, and possibly other recombinant vaccines, in C. glutamicum.
\end{abstract}

\section{Hosted file}

manuscript.doc available at https://authorea.com/users/452006/articles/550171-hypersecretionof-vaccine-antigen-outer-membrane-lipoprotein-a-in-corynebacterium-glutamicum-throughhigh-throughput-based-development-process

\section{Hosted file}

Figure.doc available at https://authorea.com/users/452006/articles/550171-hypersecretion-ofvaccine-antigen-outer-membrane-lipoprotein-a-in-corynebacterium-glutamicum-through-highthroughput-based-development-process

\section{Hosted file}

Table.doc available at https://authorea.com/users/452006/articles/550171-hypersecretion-ofvaccine-antigen-outer-membrane-lipoprotein-a-in-corynebacterium-glutamicum-through-highthroughput-based-development-process 